\title{
Time-Dependent Interactions of Oxidant-Sensitive Fluoroprobes with Inhibitors of Cellular Metabolism
}

\author{
Kirsten E. Tollefson, James Kroczynski, and Michael V. Cutaia \\ Pulmonary Disease Section, Department of Medicine, Veterans Administration Medical Center, and State University \\ of New York/Downstate Health Sciences Center, Brooklyn, New York
}

\begin{abstract}
SUMMARY: We tested three oxidant sensitive fluoroprobes (dihydrorhodamine [DHR], 2',7'-dichlorodihydrofluorescein [H $\left.{ }_{2} D C F\right]$, and dihydroethidium [DHE]) for interactions with three inhibitors of mitochondrial electron transport. DHR, $\mathrm{H}_{2} \mathrm{DCF}$, and $\mathrm{DHE}$ produced large time-dependent increases in fluorescence in a cell-free medium that contained either of the site III inhibitors antimycin (A) and 2-heptyl-4-hydroxy-quinoline-N-oxide but minimal increases in medium that contained another site III inhibitor, myxothiazol (Mx). The interactions between $A$ and each of the fluoroprobes occurred at concentrations of agent/probe that are frequently used in experiments designed to investigate cellular oxidant production. To define more effectively the nature of these agent/probe interactions, we determined the oxygen dependence of the interactions between $A$ and each probe. $T$ he $A / H_{2} D C F$ and A/DHR interactions either were highly oxygen-dependent or exhibited a small degree of oxygen dependence, respectively, whereas the A/DHE interaction was oxygen-independent. Finally, we determined multiple ways to reduce the impact of the agent/probe interaction on data acquisition. The addition of either fetal bovine serum (10\%) or albumin (5\%) to the media abolished the $A / D H R$ and $A / H_{2}$ DCF interactions. Shifting the excitation wavelength of DHE (from 470 to $530 \mathrm{~nm}$ ) reduced measurement of the A/DHE interaction while preserving measurement of the intracellular signal. Collectively, these results emphasize the importance of testing for interactions between agents and probes, because these interactions can interfere with the accurate interpretation of experimental results. In addition, the methods presented for circumventing these interactions may be applicable to other experiments in which agent/probe interactions are an obstacle to accurate interpretation of the experimental results. (Lab Invest 2003, 83:367-375).
\end{abstract}

$T$ he role of reactive oxygen species (ROS) or reactive nitrogen species in normal cellular metabolism and cell injury is an active area of research. Recent work has demonstrated a link between oxidant production and signal transduction as a part of normal cell function (Lander, 1997). Oxidant production is also a part of a variety of different pathologic conditions, including cell senescence (Bindokas et al, 1996; Zhu et al, 1994), ischemia/reperfusion injury, and hypoxia/ reoxygenation (Bindokas et al, 1996; Dawson et al, 1993; Zulueta et al, 1997). Oxidants have the potential to react with many cellular molecules, including amino acids, proteins, nucleic acids, and the lipid components of the plasma membrane (Dawson et al, 1993; Lander, 1997).

Significant progress in the field of ROS/reactive nitrogen species research was made with the development of oxidant-sensitive fluoroprobes. These probes are frequently used for the measurement of oxidant production in living cells or organ systems (Al Mehdi et al, 1997; Bindokas et al, 1996; Carter et al, 1994; Dawson et al, 1993; Hempel et al, 1999; Royall and Ischiropoulos, 1993; Zulueta et al, 1997). This methodology involves the use of chemically reduced

DOI: 10.1097/01.LAB.0000059934.53602.4F

Received December 18, 2002.

This work was supported by a VA Merit Review Grant to Dr. Cutaia. Address reprint requests to: Dr. Michael Cutaia, VA Medical Center, 800 Poly Place,Brooklyn, NY 19209-7104.E-mail:michael.cutaia@med.va.gov forms of parent dyes that are typically nonfluorescent or weakly fluorescent in the absence of oxidants but that are converted to fluorescent metabolites in the presence of oxidants. Three commonly used fluoroprobes for this purpose are 2',7'-dichlorodihydrofluorescein diacetate $\left(\mathrm{H}_{2} \mathrm{DCF}-\mathrm{DA}\right)$, dihydrorhodamine 123 (DHR), and dihydroethidium (DHE). These probes have been widely used to measure oxidant production in living cells and/or isolated perfused organ systems under a variety of different circumstances using flow cytometry(Carter et al, 1994; Garcia-Ruiz et al, 2000), fluorescence microscopy (Al Mehdi et al, 1997; Bindokas et al, 1996; Hempel et al, 1999; Zulueta et al, 1997), microplate fluorimetry (Zulueta et al, 1997), and spectrofluorimetry (Dawson et al, 1993; Garcia-Ruiz et al, 2000; Royall and Ischiropoulos, 1993).

Recent work used these probes to investigate the effect of inhibition of mitochondrial electron transport on intracellular oxidant production in different cell lines: U937 cells (Brambilla and Cantoni, 1998), rat hepatocytes (Dawson et al, 1993), and cortical neurons (Dugan et al, 1995). During our own experiments in this area, we observed interactions between several of the metabolic inhibitors and the fluoroprobes. This observation highlighted the problem of a possible interaction between a probe and other reagents used in an experiment. Interactions between agents and oxidant-sensitive probes in cell culture media have been previously reported (Benov et al, 1998; Cathcart et al, 1983; Hempel et al, 1999; Kalinich et al, 1997; 
Marchesi et al, 1999; Martin et al, 1998; Rota et al, 1999; Zhu et al, 1994). This problem is particularly relevant to work with oxidant-sensitive probes because of previous work demonstrating that optimal probe retention requires the presence of the probe in the medium during the experiment (Royall and Ischiropoulos, 1993).

The present results extend previous work on agent/ probe interactions in the following ways. First, they demonstrate for the first time the presence of a time-dependent interaction between three oxidantsensitive probes and several inhibitors of the mitochondrial electron transport chain. Second, these results provide insight into the mechanisms of these interactions. Third, we present several useful approaches for minimizing these interactions in experimental protocols. The significance of testing for and attempting to reduce or eliminate these interactions is that they can lead to artifacts that are easily confused with intracellular oxidant production and interfere with the accurate interpretation of the experimental results.

\section{Results}

The addition of antimycin (A, $20 \mu \mathrm{g} / \mathrm{mL})$ and 2-deoxyD-glucose (D, $10 \mathrm{~mm})$ to cell-free medium that contained DHR $(1 \mu \mathrm{M})$ produced a large increase in medium fluorescence over 2 hours (Fig. 1). The fluorescent signal increased gradually over the course of the experiment and did not reach a plateau at the end of the 2-hour measurement period. In contrast, there was no increase in fluorescence in cell-free media that contained DHR $(1 \mu \mathrm{M})$ in the absence of $A / D$, demonstrating that the interaction between the metabolic inhibitor(s) and the probe occurred in the medium. In the absence of $D$, cell-free media that contained $A$ and DHR demonstrated the same response as illustrated in Figure 1 (data not shown). Similarly, in the absence of $A / D$, there was no increase in fluorescence in DHR-loaded cells. The magnitude of the increase in medium fluorescence in medium that contained DHR,

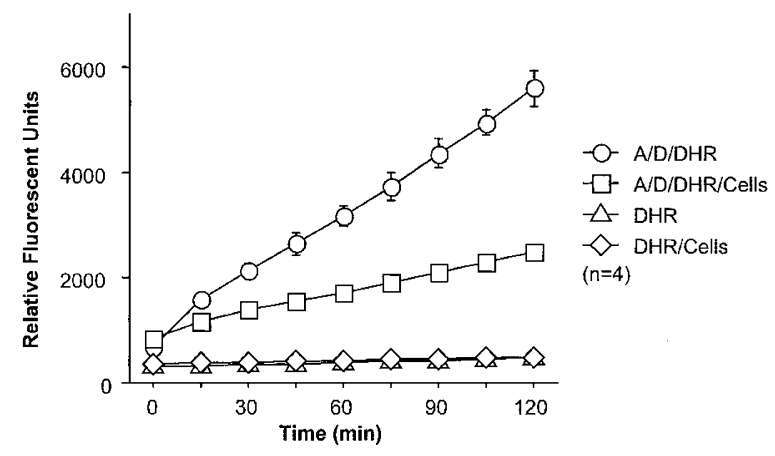

Figure 1.

Antimycin (A) interacts with DHR in cell-free media, producing a timedependent increase in fluorescence. Dihydrorhodamine (DHR; $1 \mu \mathrm{m}$ ) was added to minimal essential media (MEM) in the absence or presence of $\mathrm{A} / 2$-deoxy-D-glucose ( $\mathrm{D} ; 20 \mu \mathrm{g} / \mathrm{ml} ; 10 \mathrm{~mm}$ ); the solutions were then added to the wells of a 96-well plate as cell-free media or to wells that contained media and confluent human pulmonary artery endothelial cells; $n=6$ wells; fluorescence was monitored for 2 hours at excitation/emission wavelengths of $485 \mathrm{~nm} / 530 \mathrm{~nm}$, respectively.
DHR-loaded cells, and A/D was $\sim 50 \%$ of the increase observed in cell-free medium that contained DHR and $A / D$. Figure 2 illustrates the effects of varying doses of A $(0.1$ to $20 \mu \mathrm{g} / \mathrm{ml})$ with a fixed dose of $D(10 \mathrm{~mm})$ on fluorescence in cell-free media in the presence of DHR. There was an increase in the magnitude of media fluorescence as the A concentration increased to $1 \mu \mathrm{g} / \mathrm{ml}$. There was no significant additional increase in media fluorescence with the higher doses of A.

Collectively, the data in Figures 1 and 2 demonstrate the presence of a time-dependent interaction in media that contain A and DHR. The increase in media fluorescence required the simultaneous presence of both A and DHR. These findings demonstrate that the increase in media fluorescence observed in the presence of $A / D$ and $D H R$ was not due to $A$ alone, an interaction between $A$ and some other component of the media, or spontaneous oxidation of DHR in the media. A qualitatively similar time- and dose-dependent agent/probe interaction was observed with $\mathrm{DHR}$ and 2-heptyl-4-hydroxy-quinoline-N-oxide (HQNO), another site III inhibitor (data not shown). We also observed a minimal increase in fluorescence in medium that contained DHR and another site III inhibitor, myxothiazol $(\mathrm{Mx})$ and $\mathrm{D}(10 \mu \mathrm{M} ; 10 \mathrm{~mm})$. Unlike the interaction of DHR with $A / D$, the increase in medium fluorescence observed with $\mathrm{DHR}$ and $\mathrm{Mx} / \mathrm{D}$ was minimally timedependent (data not shown).

In a similar manner, we monitored the interaction of $\mathrm{H}_{2}$ DCF-DA with the same metabolic inhibitors. There was no increase in fluorescence in cell-free medium that contained $\mathrm{H}_{2}$ DCF-DA (5 $\left.\mu \mathrm{M}\right)$ and A/D $(20 \mu \mathrm{g} / \mathrm{ml}$; $10 \mathrm{~mm}$; Fig. 3). In contrast, the active form of the probe, $\mathrm{H}_{2}$ DCF $(5 \mu \mathrm{M})$, produced a time-dependent increase in medium fluorescence in the presence of A/D $(20 \mu \mathrm{g} / \mathrm{ml} ; 10 \mathrm{~mm})$, similar to DHR. $\mathrm{H}_{2}$ DCF alone in solution demonstrated a small increase in medium fluorescence over 2 hours, suggesting a small degree of spontaneous probe oxidization during the experiment. The increase in media fluorescence with $A / D$ and $\mathrm{H}_{2} \mathrm{DCF}$ also demonstrated a dependence on the dose of $A$ (data not shown). The response of $\mathrm{H}_{2} \mathrm{DCF}$ to

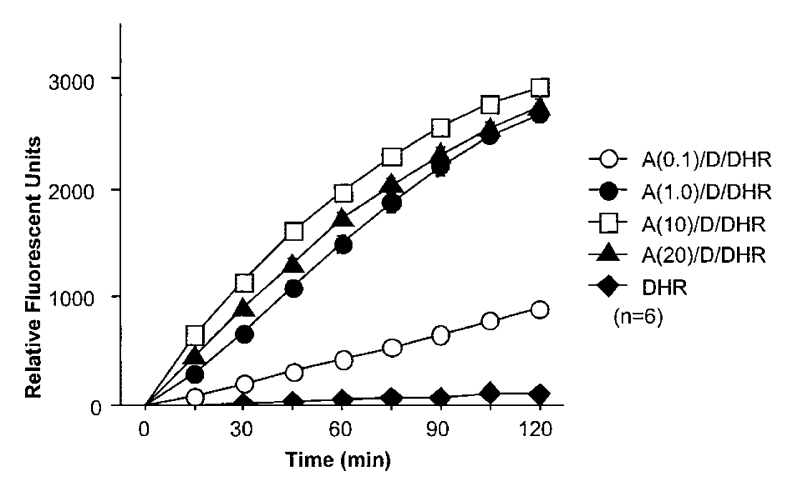

Figure 2.

The interaction of A with DHR in cell-free media is maximal at a dose of 1 $\mu \mathrm{g} / \mathrm{ml} \mathrm{A}$. DHR $(1 \mu \mathrm{M})$ was incubated with $\mathrm{D}(10 \mathrm{~mm})$ and increasing doses of A $(0.1$ to $20 \mu \mathrm{g} / \mathrm{ml}) ; n=6$ wells. 


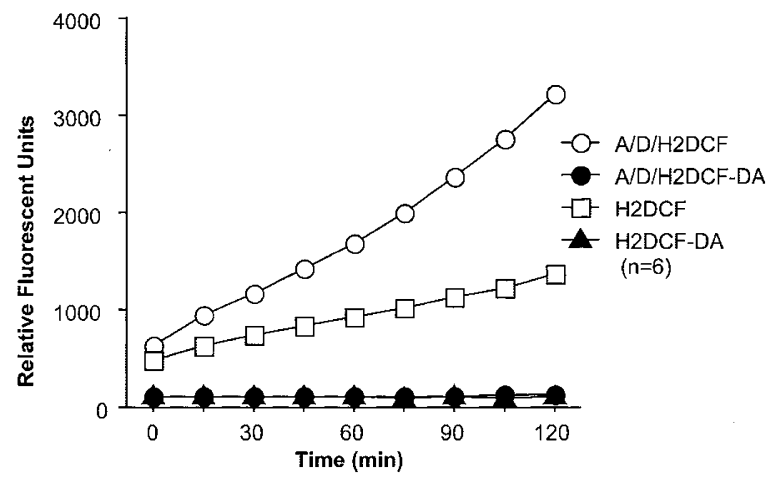

Figure 3.

Incubation of $A$ with $2^{\prime}, 7^{\prime}$-dichlorodihydrofluorescein $\left(\mathrm{H}_{2} \mathrm{DCF}\right)$ but not $\mathrm{H}_{2}$ DCF-DA increases media fluorescence. $\mathrm{H}_{2}$ DCF-DA $(5 \mu \mathrm{M})$ or its active, de-esterified form, $\mathrm{H}_{2} \mathrm{DCF}(5 \mu \mathrm{m})$, was incubated in the absence or presence of $\mathrm{A} / \mathrm{D}(20 \mu \mathrm{g} / \mathrm{ml} ; 10 \mathrm{~mm}) ; n=6$ wells; fluorescence was monitored for 2 hours at excitation/emission wavelengths of $485 \mathrm{~nm} / 530 \mathrm{~nm}$, respectively.

HQNO and Mx was qualitatively similar to the results with DHR (data not shown).

In separate experiments, we monitored the same agent/probe interactions with DHE. DHE alone in cell-free medium demonstrated a small increase in fluorescence over time, like $\mathrm{H}_{2} \mathrm{DCF}$, suggesting some spontaneous oxidation of the probe to its fluorescent metabolite, ethidium (Fig. 4). Addition of A/D (0.1 to 20 $\mu \mathrm{g} / \mathrm{ml} / 10 \mathrm{~mm}$ ) to cell-free medium that contained DHE $(5 \mu \mathrm{M})$ produced a time-dependent increase in fluorescence, similar to the pattern observed with $\mathrm{DHR}$ and $\mathrm{H}_{2}$ DCF. This response did not occur below $10 \mu \mathrm{g} / \mathrm{ml}$ of A. DHE also demonstrated similar interactions with the other metabolic inhibitors (HQNO, Mx), as observed with DHR and $\mathrm{H}_{2}$ DCF (data not shown).

DHE and DHR are used by monitoring the accumulation of their fluorescent metabolites, ethidium and rhodamine 123 , respectively, in the presence of oxidants. We determined whether the interaction of $A$ with each probe occurred with the parent probe, or the oxidized metabolite (Fig. 5). We compared the interaction of $\mathrm{A}(20 \mu \mathrm{g} / \mathrm{mL})$ with each probe (DHR, $1 \mu \mathrm{M}$; DHE, $5 \mu \mathrm{M}$ ) or with their oxidized products (R123, 1 $\mu \mathrm{M}$; EtBr, $5 \mu \mathrm{M})$, respectively. Ethidium bromide (EtBr)

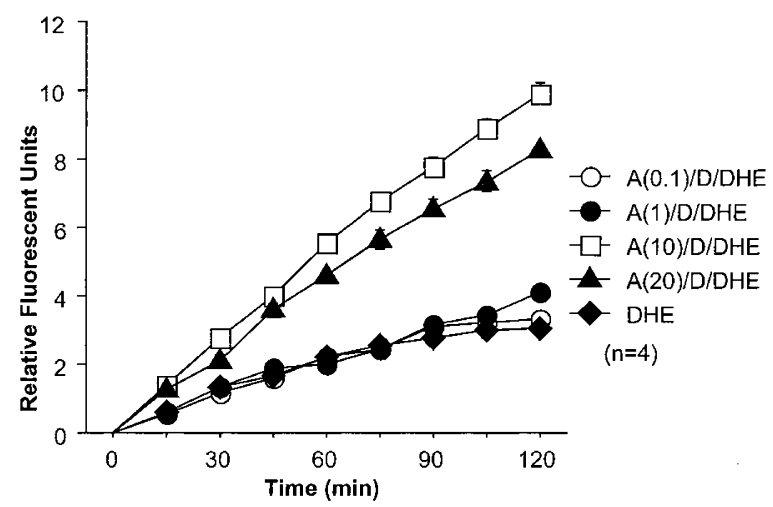

Figure 4.

Fluorescence is produced by the incubation of DHE with doses of $A$ of 10 $\mu \mathrm{g} / \mathrm{ml}$ or greater. Dihydroethidium (DHE; $5 \mu \mathrm{M}$ ) was incubated with varying doses of $\mathrm{A}(0.1$ to $20 \mu \mathrm{g} / \mathrm{ml})$ and $\mathrm{D}(10 \mathrm{~mm}) ; n=4$ wells.

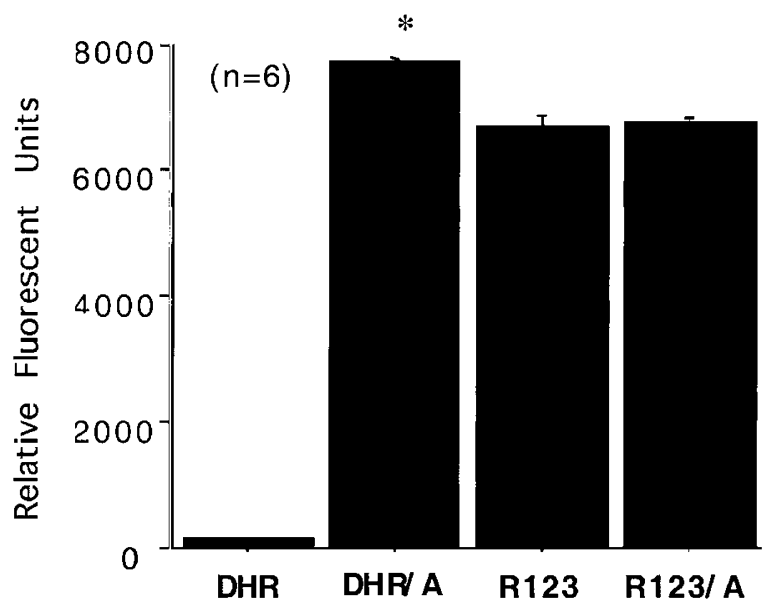

Experimental Conditions

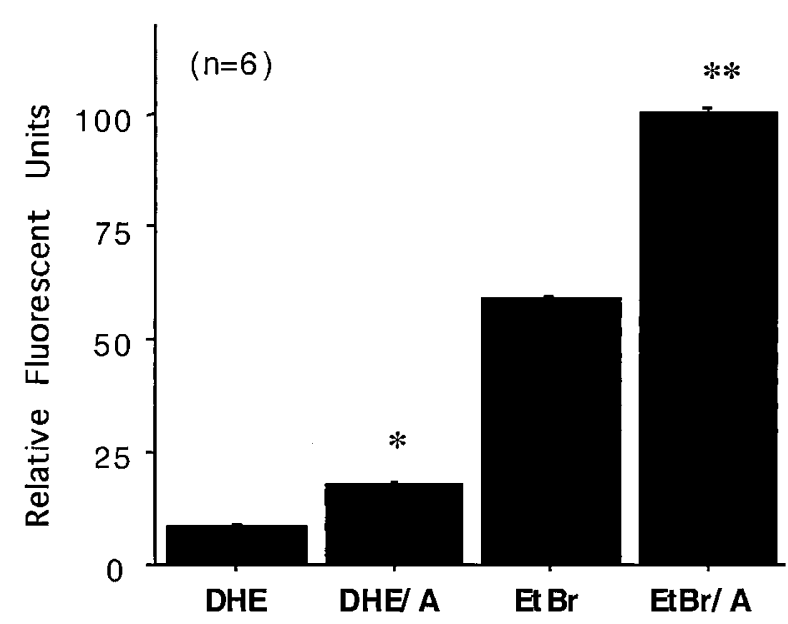

Experimental Conditions

Figure 5.

A interacts with DHR and with the product of DHE oxidation (ethidium). A, DHR $(1 \mu \mathrm{M})$ and R123 $(1 \mu \mathrm{M})$ were incubated in the absence or presence of A (20 $\mu \mathrm{g} / \mathrm{ml}) ; n=6$ wells; fluorescence was monitored for 2 hours at excitation/ emission wavelengths of $485 \mathrm{~nm} / 530 \mathrm{~nm}$, respectively; ${ }^{*} p<0.001$ versus DHR. B, DHE $(5 \mu \mathrm{M})$ and ethidium bromide $(\mathrm{EtBr} ; 5 \mu \mathrm{M})$ were incubated in the absence or presence of $A(20 \mu \mathrm{g} / \mathrm{ml}) ; n=6$ wells; fluorescence was monitored for 2 hours at excitation/emission wavelengths of $470 \mathrm{~nm} / 610 \mathrm{~nm}$, respectively; ${ }^{*} p<0.001$ versus DHE; ${ }^{* \star} p<0.001$ versus EtBr.

was chosen to represent the oxidized molecule of DHE because it is a readily available form of ethidium. Incubation of R123 with A in cell-free medium for 2 hours produced no change in media fluorescence, compared with R123 alone. In contrast, incubation of $\mathrm{DHR}$ with A led to a 40-fold increase in the signal over the level observed with DHR alone (Fig. 5A), suggesting that the increase in medium fluorescence is a direct result of an interaction between DHR and A. In contrast, medium fluorescence increased $\sim 100 \%$ in the presence of $\mathrm{A}$ and either $\mathrm{DHE}$ or $\mathrm{EtBr}$, compared 
with the signal obtained with DHE or EtBr alone (Fig. $5 B$ ). The wavelength settings in this experiment were set to monitor only generation of ethidium. Therefore, these results suggest that the major source of media fluorescence is the interaction of $A$ with ethidium.

The oxygen dependence of the agent/probe interactions was determined by incubating each probe for 1 hour under normoxic or hypoxic conditions in the absence or presence of $A$, followed by measurement of the magnitude of the signal in the cell-free medium under each condition (Table 1). Under normoxic conditions, agent/probe interactions were again evident, noted by the increase in media fluorescence in normoxic cell-free media that contained A/probe above the level observed in media that contained probe alone. Spontaneous oxidation of $\mathrm{H}_{2}$ DCF and DHE was significantly reduced in hypoxic medium, noted by the decrease in the magnitude of the fluorescent signal under these conditions, compared with signal in normoxic medium containing probe. The agent/probe interaction with $A$ and $\mathrm{H}_{2}$ DCF was abolished under hypoxic conditions, as indicated by the lack of a significant increase in media fluorescence in hypoxic media that contained $\mathrm{A} /$ probe, compared with media that contained probe alone. Similarly, the agent/probe interaction with A/DHR was greatly attenuated under hypoxic conditions. There was only a small $(\sim 31 \%)$ increase in medium fluorescence in hypoxic medium that contained A/DHR, compared with medium that contained DHR alone. In contrast, under normoxic conditions, A/DHR produced a $131 \%$ increase in media fluorescence, compared with media that contained DHR alone. In contrast to the oxygen dependence of the interactions of $A$ with either $\mathrm{H}_{2} \mathrm{DCF}$ or DHR, the A/DHE interaction was oxygen-independent. There was a similar increase in media fluorescence under both normoxic and hypoxic conditions.

We investigated several ways to reduce or eliminate agent/probe interactions. Addition of varying concentrations of either fetal bovine serum (5\%, 10\%) or albumin (5\%) to cell-free media that contained A/D (20 $\mu \mathrm{g} / \mathrm{ml} ; 10 \mathrm{~mm})$ and either DHR $(1 \mu \mathrm{M})$ or $\mathrm{H}_{2} \mathrm{DCF}(5 \mu \mathrm{M})$ markedly reduced media fluorescence (Fig. 6). Albumin was more effective in reducing medium fluorescence with DHR than FBS (Fig. 6A). Both FBS (10\%) and albumin $(5 \%)$ reduced media fluorescence below the level observed with $\mathrm{H}_{2}$ DCF alone (Fig. 6B).
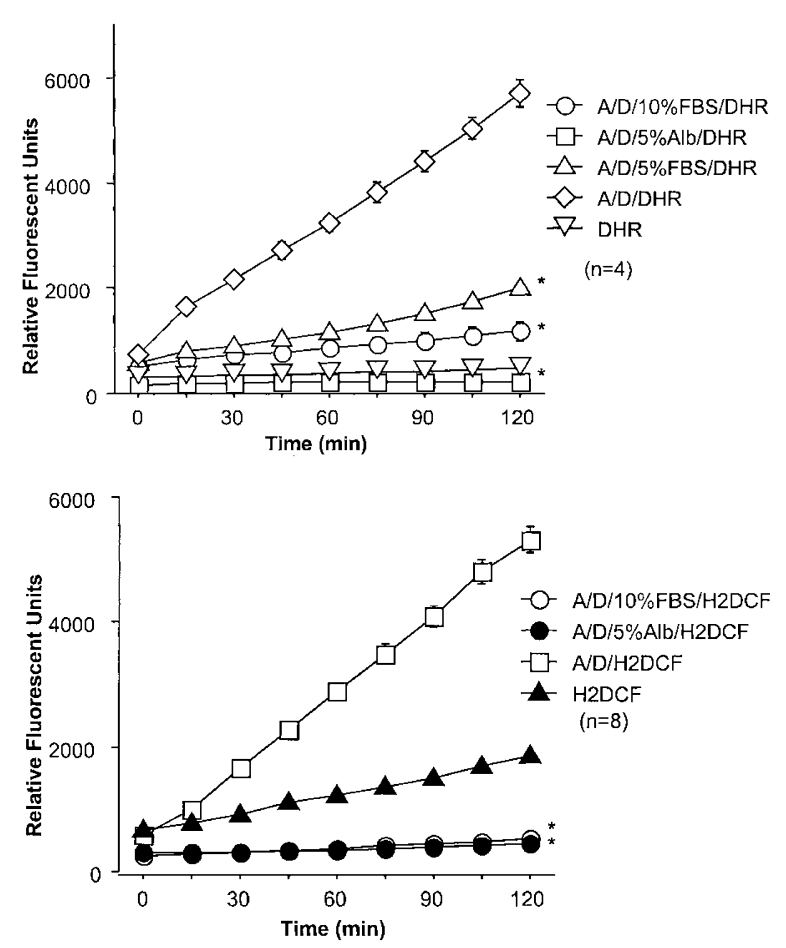

Figure 6.

FBS and albumin inhibit the interaction between $A$ and DHR or $\mathrm{H}_{2} \mathrm{DCF}$. A, DHR $(1 \mu \mathrm{M})$ and $\mathrm{A} / \mathrm{D}(20 \mu \mathrm{g} / \mathrm{ml} ; 10 \mathrm{~mm})$ were incubated in MEM that contained $5 \%$ to $10 \%$ FBS or $5 \%$ albumin; $n=4$ wells; ${ }^{\star} p<0.001$ versus DHR/A/D. B, $\mathrm{H}_{2}$ DCF $(5 \mu \mathrm{M})$ and $\mathrm{A} / \mathrm{D}(20 \mu \mathrm{g} / \mathrm{ml} ; 10 \mathrm{~mm})$ were incubated in MEM that contained $10 \%$ FBS, or in the presence of $5 \%$ albumin; $n=4$ wells; fluorescence was monitored for 2 hours at excitation/emission wavelengths of $485 \mathrm{~nm} / 530 \mathrm{~nm}$, respectively; * $p<0.001$ versus $\mathrm{H}_{2} \mathrm{DCF} / \mathrm{A} / \mathrm{D}$.

The second approach to reducing agent/probe interactions involved adjusting the excitation settings used with DHE. Investigators have used several different excitation wavelengths (475 to $530 \mathrm{~nm}$ ) with an emission wavelength at $610 \mathrm{~nm}$ when using this probe (Al Mehdi et al, 1997; Benov et al, 1998; Bindokas et al, 1996; Carter et al, 1994). Therefore, we performed a series of excitation/emission scans to determine the optimal excitation settings in our hands. The emission scan always demonstrated a broad peak at $\sim 610 \mathrm{~nm}$ (data not shown). Therefore, this wavelength setting was not altered; all excitation scans were performed using an emission wavelength of $610 \mathrm{~nm}$. In contrast, the excitation scans revealed differences in the spec-

Table 1. The A/Probe Interaction Is Oxygen-Dependent for $\mathrm{H}_{2} \mathrm{DCF}$ and DHR but 0 xygen-Independent for DHE

\begin{tabular}{lccccc}
\hline & $\begin{array}{c}\text { Normoxia } \\
\text { (Media alone) }\end{array}$ & $\begin{array}{c}\text { Normoxia } \\
\text { (Probe) }\end{array}$ & $\begin{array}{c}\text { Normoxia } \\
\text { (A/Probe) }\end{array}$ & $\begin{array}{c}\text { Hypoxia } \\
\text { (Probe) }\end{array}$ & $\begin{array}{c}\text { Hypoxia } \\
(\text { A/Probe })\end{array}$ \\
\hline $\mathrm{H}_{2}$ DCF: $(5 \mu \mathrm{M})$ & $3.0 \pm 0.1$ & $100.5 \pm 3.3$ & $178.1 \pm 0.2^{a}$ & $46.4 \pm 2.4^{b}$ & $37.9 \pm 0.6$ \\
DHR: $(1 \mu \mathrm{M})$ & $10.9 \pm 0.2$ & $62.2 \pm 0.7$ & $142.9 \pm 5.5^{a}$ & $55.1 \pm 1.1$ & $72.3 \pm 1.7^{c}$ \\
DHE: $(5 \mu \mathrm{M})$ & $1.4 \pm 0.03$ & $2.9 \pm 0.02$ & $3.8 \pm 0.04^{a}$ & $2.0 \pm 0.02^{b}$ & $3.0 \pm 0.05^{c}$ \\
\hline
\end{tabular}

Fluorescence was monitored after a 1-hour incubation under the following experimental conditions. Normoxia, solutions that contained probe or A/probe exposed to room air; hypoxia, solutions that contained probe or A/probe bubbled with argon before and during incubation; the data are expressed in relative fluorescent units under each experimental condition; wavelengths settings for each probe: $\mathrm{H}_{2}$ DCF-DA, DHR, excitation $485 \mathrm{~nm} / \mathrm{emission} 530 \mathrm{~nm}$; DHE, excitation $470 \mathrm{~nm} / \mathrm{emission} 610$ $\mathrm{nm} ; n=5-6$ wells of a 96-well plate for each condition.

${ }^{a} p<0.0001$ versus normoxia (probe).

${ }^{b} p<0.0001$ versus normoxia (probe).

${ }^{c} p<0.0001$ versus hypoxia (probe). 
tral characteristics of DHE in medium alone versus when DHE was loaded into cells (Fig. 7A). DHE alone in solution demonstrated a broad peak extending from excitation wavelengths 460 to $520 \mathrm{~nm}$. In cell-free medium that contained $\mathrm{DHE}$ and $\mathrm{A}$, there was a similar peak at wavelengths 460 to $520 \mathrm{~nm}$, which represented $\sim 100 \%$ increase in the magnitude of the signal with DHE alone. The difference between the signals under these conditions represented the agent/probe interaction. The magnitude of this difference in signal
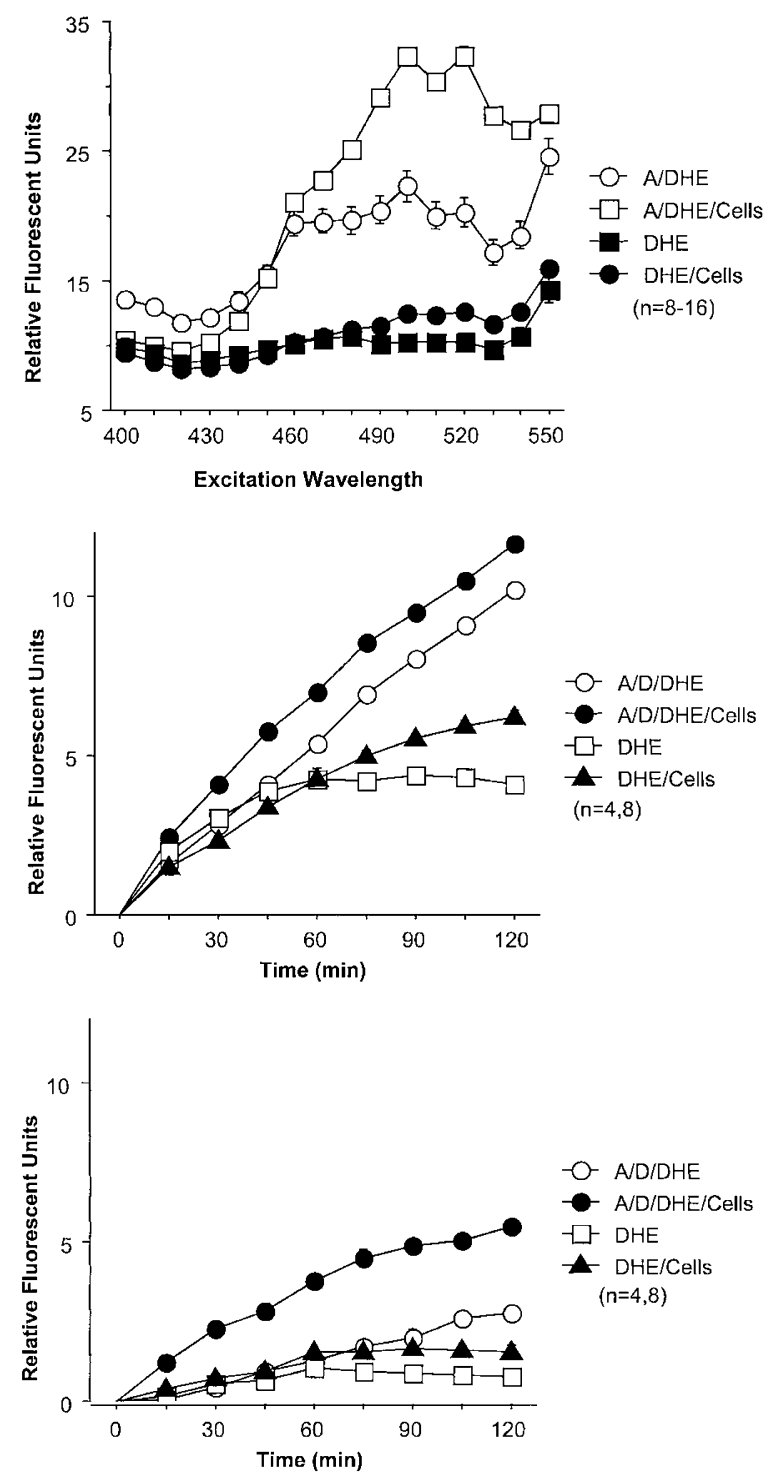

Figure 7.

Altering the excitation wavelength of DHE reduces the contribution of media fluorescence to the measured fluorescent signal. A, Excitation scans over the range of 400 to $550 \mathrm{~nm}$ were performed after a 2-hour incubation of DHE (5 $\mu \mathrm{M})$ in the absence or presence of $A / D(20 \mu \mathrm{g} / \mathrm{ml} ; 10 \mathrm{~mm})$, either in cell-free media or in DHE-loaded cells; fluorescence was monitored at an emission wavelength of $610 \mathrm{~nm} ; n=4$ wells. B and C, DHE (5 $\mu \mathrm{m})$ was incubated for 2 hours in the absence or presence of $A / D(20 \mu \mathrm{g} / \mathrm{ml} ; 10 \mathrm{~mm})$, either in cell-free media or in DHE-loaded cells; fluorescence was monitored at two excitation wavelengths: either $470 \mathrm{~nm}$ or $530 \mathrm{~nm}$ for B or C, respectively, with an emission wavelength of $610 \mathrm{~nm} ; n=4$ to 8 wells. strength (DHE alone versus A/DHE) became smaller at excitation wavelengths $>520 \mathrm{~nm}$.

In contrast to the results with cell-free media, DHEloaded cells demonstrated a more narrow range for the peak signal, which extended from excitation wavelengths 500 to $520 \mathrm{~nm}$. The magnitude of this signal was larger than the signal observed in cell-free medium that contained DHE. DHE-loaded cells incubated with A/D $(20 \mu \mathrm{g} / \mathrm{ml} ; 10 \mathrm{~mm})$ also demonstrated this same narrow peak signal range (500 to $520 \mathrm{~nm}$ ). The presence of cells significantly increased the magnitude of the signal in the 500- to 520-nm range, indicating that the signal at these higher wavelengths represented the signal from the intracellular compartment. This signal represents endogenous production secondary to inhibition of mitochondrial electron transport at site III (Dawson et al, 1993; Garcia-Ruiz et al, 2000; Turrens, 1997). Thus, use of an excitation setting $>500 \mathrm{~nm}$ was associated with a significant decrease in the proportion of the signal that represented agent/probe interaction in the media.

We confirmed these findings by measuring the increase in signal strength under the same conditions and concurrently monitoring signal strength at two different excitation settings (470 versus $530 \mathrm{~nm}$ ) at an emission of $610 \mathrm{~nm}$. At an excitation wavelength of $470 \mathrm{~nm}$, we again observed a time-dependent increase in medium fluorescence when $A / D$ was added to $\mathrm{DHE}$ in cell-free medium. The A/D/DHE signal in the presence of cells was $\sim 10 \%$ larger than the signal obtained with DHE alone in cell-free medium (Fig. 7B). In contrast, using an excitation setting of $530 \mathrm{~nm}$, the A/D/DHE signal in the presence of cells was $\sim 100 \%$ larger than the A/D/DHE signal in cell-free medium (Fig. 7C). Collectively, these results demonstrate that the DHE assay can be optimized for measurement of intracellular oxidant production using an excitation wavelength of $530 \mathrm{~nm}$. This approach reduces the component of the signal representing medium fluorescence secondary to agent/probe interaction.

\section{Discussion}

In this study, we evaluated three commonly used oxidant-sensitive probes for interactions with several metabolic inhibitors. DHR, $\mathrm{H}_{2} \mathrm{DCF}$, and DHE demonstrated a significant time-dependent interaction with several mitochondrial electron transport chain inhibitors that are frequently used in experiments designed to investigate altered cell function (Brambilla and Cantoni, 1998; Dawson et al, 1993; Turrens, 1997). To our knowledge, this is the first demonstration of timedependent agent/probe interactions between metabolic inhibitors and oxidant-sensitive probes, leading to the production of a fluorescent signal in the cell medium. The significance of these agent/probe interactions is that these artifacts could be confused with the signals generated in response to intracellular oxidant production. Different types of interactions were observed between the same agent and different probes, highlighting the need to define carefully the nature of these interactions for different combinations 
of agents and probes in a specific experimental protocol. Finally, we demonstrated several useful ways to reduce or eliminate these agent/probe interactions. These methods include the addition of protein to the media and the modification of the excitation wavelength settings with a specific probe (DHE). These modifications permit more effective monitoring of the intracellular signals generated in response to stimuli known to increase intracellular oxidant production.

Several authors have described time-dependent interactions between chemical agents and oxidantsensitive probes (Hempel et al, 1999; Kalinich et al, 1997; Martin et al, 1998; Zhu et al, 1994). One of the first reports of a time-dependent interaction was the oxidation of $\mathrm{H}_{2}$ DCF by xanthine oxidase (Zhu et al, 1994). In this study, xanthine oxidase oxidized the probe to its fluorescent form in the absence of ROS production. Subsequent work demonstrated that Trolox and chromate $\mathrm{V}$ enhanced the oxidation of $\mathrm{H}_{2}$ DCF to DCF and that the enhancement as a result of Trolox could be prevented by the addition of other antioxidants to the experimental media (Kalinich et al, 1997; Martin et al, 1998). Hempel et al (1999) recently demonstrated that $\mathrm{Cu} / \mathrm{Zn}-\mathrm{SOD}$ oxidized multiple ROS probes, including $\mathrm{H}_{2} \mathrm{DCF}$ and $\mathrm{DHR}$, and could act as a cofactor in the oxidation of these probes by $\mathrm{H}_{2} \mathrm{O}_{2}$. $\mathrm{H}_{2}$ DCF and DHR also demonstrated a timedependent interaction with several other compounds, including horseradish peroxidase, ferric iron, and cytochrome $\mathrm{C}$ in this study.

Other types of artifacts have also been reported with $\mathrm{H}_{2}$ DCF. Rota et al (1999) demonstrated that, in the presence of horseradish peroxidase and a reducing agent, $\mathrm{H}_{2}$ DCF cycles between an oxidized phenol radical form and its reduced form, generating superoxide anion and consuming oxygen. Earlier work from the same laboratory suggested that this process was triggered by light exposure of $\mathrm{H}_{2} \mathrm{DCF}$ (Marchesi et al, 1999). The significance of these types of interactions is that they can result in the generation of an exaggerated $\mathrm{H}_{2}$ DCF response in an experimental system under investigation. We did not encounter this type of light-dependent artifact in our work, as evidenced by the absence of an increase in experiments that used cell-free media that contained a probe but no metabolic inhibitors. The present experiments were performed under conditions in which we took special precautions to minimize light exposure.

Much less is known about interactions between DHE and biochemical agents under different experimental conditions. Bindokas et al (1996) found that DHE may be a weak substrate for xanthine oxidase, like $\mathrm{H}_{2}$ DCF (Zhu et al, 1994), but the magnitude of the interaction was small. DHE is oxidized by cytochrome c, an agent that also oxidizes DHR and $\mathrm{H}_{2}$ DCF (Benov et al, 1998; Rota et al, 1999). In contrast, DHE is not oxidized by certain of the agents that oxidize $\mathrm{H}_{2}$ DCF and DHR, such as horseradish peroxidase, catalase, and allopurinol (Carter et al, 1994). These findings emphasize the differential reactivity of probes to different reagents and, thus, the specificity of agent/ probe interactions.
The present results documented differences between the response of DHE to metabolic inhibitors versus the response of $\mathrm{DHR}$ and $\mathrm{H}_{2} \mathrm{DCF}$ to these same agents. One example is the nature of the interaction between A and DHR or DHE. An agent could interact directly with a probe or with the active metabolite of the probe. The results in Figure 5 demonstrate that $A$ reacts with $\mathrm{DHR}$ but not with its active metabolite, R123. In contrast, the nature of the interaction between $A$ and DHE is different. The present results suggest that $A$ interacts with the active, oxidized metabolite of DHE (ethidium). These findings demonstrate the heterogeneity of agent/probe interactions. In addition, the A/DHE interaction was still observed under hypoxic conditions (oxygen-independent), in contrast to the interaction of $A$ with DHR or $\mathrm{H}_{2} \mathrm{DCF}$. These agent/probe interactions demonstrated a mild to marked degree of oxygen dependence, respectively. Thus, the type of interaction between a specific agent and different probes cannot be assumed to be identical. These findings demonstrate the specificity of agent/probe interactions. The present results do not provide insight into the mechanism of specific agent/ probe interactions at the molecular level.

Collectively, the present results and previous work on agent/probe interactions demonstrate the diversity of interactions (and potential artifacts) that can occur in experiments that involve the use of different fluoroprobes. These findings illustrate the need for thorough testing for possible agent/probe interactions in an experimental protocol.

We have found that a useful approach to detect and monitor agent/probe interactions is to design experiments so that the fluorescent signal can be measured in both cells and cell-free media simultaneously. This can easily be done when working with cultured cells grown in tissue culture plates where a plate can be partitioned into sections that contain only cell-free media versus media plus cells. This technique allows the investigator to recognize agent probe/interactions more readily, quantify the magnitude of these interactions, and test the utility of corrective actions based on knowledge of the spectral characteristics of the agent/ probe interaction.

The type of interaction observed in the media between $\mathrm{A}$ and DHE would have been especially difficult to analyze and circumvent if we had not used the approach of optimizing the excitation wavelength setting used with this probe. Previous work with this probe used a range of different excitation wavelength settings (470 to $535 \mathrm{~nm}$ ) (Al Mehdi et al, 1997; Benov et al, 1998; Bindokas et al, 1996; Dawson et al, 1993). The variability in the settings used with this probe highlights the variability that arises when using a specific probe under different experimental conditions and with different optical instruments. Therefore, it is important for investigators to optimize the settings used with a probe under the specific experimental conditions and on the equipment used in their laboratory. We used a monochromator-based microplate fluorimeter for the majority of our experiments. Alteration of excitation/emission wavelengths is easily 
done with this type of instrument. Using this approach, we characterized the spectral range of the medium fluorescence secondary to the interaction of $A$ with $\mathrm{DHE}$ in cell-free medium. Once this range was defined, we were able to compare the magnitude of the media fluorescence at different excitation wavelengths in the presence and absence of cells. This approach allowed selection of an excitation wavelength $(530 \mathrm{~nm})$ at which the medium fluorescence secondary to the agent/probe interaction was minimized, while the intracellular signal was well preserved. Our choice of an excitation wavelength of $530 \mathrm{~nm}$ was practical in that this is a standard wavelength for which excitation filters are commercially available for more traditional microplate fluorimeters. Therefore, this corrective measure could also be used with microplate fluorimeters that have filters instead of monochromators.

These results demonstrate the utility of optimizing the excitation/emission wavelength settings as an approach to reduce or eliminate agent/probe interactions. This approach allows investigators to define the spectral range of the signal arising from an agent/ probe interaction and consider alternative excitation settings. Optimization of excitation/emission wavelength settings is also an alternative to the technique of adding protein or albumin to the media to reduce interactions. Addition of reagents to the media might potentially complicate an experimental protocol. Nevertheless, both approaches are feasible, because they are applicable to any experiment using a fluoroprobe.

The use of fluoroprobes in molecular and cell biology continues to expand at a rapid rate. In particular, oxidant-sensitive probes continue to be used widely in different experimental settings to measure intracellular oxidant production in mammalian cells. Fluoroprobes offer significant advantages to investigators, including the avoidance of radioactive waste and the possibility of making semiquantitative measurements in living cells or organ systems. Although these advantages are increasingly recognized, the problems in achieving optimal use of these valuable tools are sometimes overlooked. The present results highlight the importance of testing for agent/probe interactions that could complicate the interpretation of experiments using fluoroprobes. Investigators should always consider the possibility of time-dependent agent/probe interactions as noted in this study and other recent work (Hempel et al, 1999; Kalinich et al, 1997; Martin et al 1998; Zhu et al, 1994). The techniques for eliminating or reducing agent/probe interactions in the present work are potentially applicable to all experiments using fluoroprobes.

\section{Materials and Methods}

\section{Cell Culture and Reagents}

Large-vessel human pulmonary artery endothelial cells were obtained from a commercial vendor (Clonetics/BioWhittaker, Walkersville, MD) and grown in endothelial cell growth medium (EGM-II) that contained $10 \%$ fetal bovine serum, as previously de- scribed (Cutaia et al, 2000). In preparation for microplate fluorimetry experiments, cells were seeded uniformly into the first eight columns of 96-well tissue culture plates using an Eppendorf repeater pipette (100 $\mu$ l per well). All experiments were conducted with cells matched for cell line, passage number, time to confluence, and cell density between experimental groups. No differences were observed in the results using different cell lines or passage numbers (5 to 18).

\section{Fluoroprobes}

We used three probes commonly used to measure oxidant production: $\mathrm{H}_{2}$ DCF-DA $(5 \mu \mathrm{M})$, DHR (1 $\left.\mu \mathrm{M}\right)$, and DHE $(5 \mu \mathrm{M})$. Each probe was used in standard manner, as previously described (Bindokas et al, 1996; Carter et al, 1994; Garcia-Ruiz et al, 2000; Royall and Ischiropoulos, 1993). $\mathrm{H}_{2}$ DCF-DA is a cellpermeant fluorescein derivative, which becomes trapped in the cell after de-esterification by intracellular esterases to form the relatively impermeant nonfluorescent molecule, $\mathrm{H}_{2}$ DCF. $\mathrm{H}_{2}$ DCF can then react with a variety of different radical species to form the highly fluorescent metabolite dichlorofluorescein. DHR is a reduced form of rhodamine 123 (R123) that readily crosses cell membranes. In the presence of oxidants, DHR is oxidized to R123, which is then sequestered in mitochondria (Royall and Ischiropoulos, 1993). DHE is a cell-permeable probe that is converted to the cell impermeant metabolite, ethidium, in the presence of oxidants (Haugland, 1996). After loading of each probe into cells, the experiments were run with the probe in the medium, based on previous work demonstrating that this approach optimizes probe retention in the cells during an experiment (Royall and Ischiropoulos, 1993).

Several experiments were performed using the active intracellular form of $\mathrm{H}_{2}$ DCF-DA. Before such experiments, $\mathrm{H}_{2}$ DCF-DA was cleaved to its fluorescent metabolite $\left(\mathrm{H}_{2} \mathrm{DCF}\right)$, using a procedure derived from the manufacturer (Molecular Probes, Eugene, OR). Briefly, the procedure involves cleaving off the diacetate moiety, normally performed by intracellular esterases, using $\mathrm{NaOH}$. This procedure consists of adding $\mathrm{MeOH}(50 \mu \mathrm{l})$ and $2 \mathrm{~N} \mathrm{NaOH}(25 \mu \mathrm{l})$ to $50 \mu \mathrm{l}$ of an $\mathrm{H}_{2}$ DCF-DA (5 mM) stock solution. The solution is then stored at room temperature in the dark for 1 to 2 hours and diluted in minimal essential media (MEM; defined below) to achieve a final working solution with a probe concentration of $5 \mu \mathrm{m}$. The $\mathrm{pH}$ of the solution is titrated to 7.4 by the addition of $5 \mathrm{~N} \mathrm{HCl}$ immediately before use.

The following wavelength settings were used to monitor the oxidized fluorescent metabolite of each probe: $\mathrm{H}_{2}$ DCF-DA, excitation $485 \mathrm{~nm} /$ emission 530 nm; DHR, excitation 485 nm/emission 530 nm; DHE, excitation $470 \mathrm{~nm}$ or $530 \mathrm{~nm} / \mathrm{emission} 610 \mathrm{~nm}$. All solutions were made fresh daily, and those that contained fluoroprobes were protected from light exposure during the experiment. 


\section{Metabolic Inhibition}

$A$ is a metabolic inhibitor that inhibits the electron transport chain at the cytochrome bc1-complex (site III). The commonly used preparation of $A$ is a mixture of four different subtypes, which differ in the length of the alkyl substituents on the dilactone ring of the molecule (Slater, 1973). Similar to A, HQNO also acts at the cytochrome bc1 complex of site III of the electron transport chain (Brambilla and Cantoni, 1998). Myxothiazol blocks the oxidation of ubiquinol at the Rieske iron-sulfur center of complex III (Dawson et al, 1993).

When A, HQNO, or Mx was used, D, a glycolysis inhibitor, was also included to simulate a standard metabolic inhibition protocol consisting of inhibition of oxidative phosphorylation and glycolysis. The exceptions were several single time point experiments in which we evaluated the spectral characteristics of an agent/probe interaction, when it was advantageous to isolate the effects of one agent on the probe. In preliminary experiments, we found that the presence of $D$ did not significantly modify any of the agent/ probe interactions within this study (data not shown).

\section{Experiments Using Cell-Free Media}

Experiments were performed in 96-well plates in HEPES-buffered MEM (116.4 mM NaCl, $5 \mathrm{~mm} \mathrm{KCl,} 0.8$ $\mathrm{mm} \mathrm{MgSO}_{4}, 5.5 \mathrm{~mm}$ glucose, $1.8 \mathrm{~mm} \mathrm{CaCl}, 1.01 \mathrm{~mm}$ $\mathrm{NaH}_{2} \mathrm{PO}_{4} 1 \mathrm{H}_{2} \mathrm{O}$, and $10 \mathrm{~mm}$ HEPES), $\mathrm{pH} 7.4$, unless otherwise specified. One plate was considered one experiment, and one well was considered a single sample (an $n$ of 1). All experiments were performed at least twice with qualitatively similar results. This approach is particularly important in view of the normal day-to-day variation in the magnitude of the fluorescent probe signal strength between experiments. Experiments were initiated by adding the fluoroprobe to the solution, followed by monitoring of the fluorescent signal. With DHR, $\mathrm{H}_{2} \mathrm{DCF}-\mathrm{DA}$, and DHE, fluoroprobes were added to the solutions last, and solutions were immediately transferred to the microplate for fluorescence monitoring. $\mathrm{H}_{2} \mathrm{DCF}$, however, was handled slightly differently because the stock was highly basic. After cleavage of the diacetate moiety, the solution was titrated to $\mathrm{pH}$ 7.4. The metabolic inhibitor was then added, the solutions were immediately transferred to the microplate, and fluorescence monitoring was begun.

In experiments performed with reagents and probes in cell-free media, media fluorescence was monitored at 15-minute intervals over 2 hours. Two different microplate fluorimeters were used in these experiments: (a) Gemini SpectraMax-2 (Molecular Devices, Menlo Park, CA) and (b) Cambridge Technologies microplate reader (Model 7630). The majority of experiments were performed on the SpectraMax because of the advantageous features of this instrument (described below). There were no qualitative differences in the results in selected experiments with either instrument.
We also performed a series of experiments designed to optimize the excitation/emission wavelength settings used with DHE. These experiments consisted of a series of excitation or emission scans on a 96-well plate, using media with DHE or A/DHE in both cell-free wells, and wells that contained DHE-loaded cells. These scans were run on the monochromator-based microplate reader (Gemini SpectraMax; Molecular Devices). The scans measured the magnitude of fluorescent signals over a range of excitation or emission wavelengths, respectively. For example, an excitation scan performed at a fixed emission wavelength was used to determine the spectral characteristics of the interaction of agents with DHE. This type of scan is commonly used to determine the range and magnitude of a fluorescent signal obtained under different conditions. This is a standard method used to optimize wavelength settings for a particular probe. These scans could be performed only with the use of a monochromator-based instrument.

Experiments designed to determine the effect of hypoxia on agent/probe interactions were conducted in the following way. Hypoxic conditions were created by bubbling MEM with argon gas ( $0 \%$ oxygen) in a covered plastic or glass tube for 30 minutes to remove oxygen from the solutions. Measurements with a dissolved oxygen probe (Lazar Research Labs, Inc., CA) demonstrated an oxygen gas content of $0.2 \%$ in the solutions bubbled with argon gas versus $21.3 \%$ in solutions exposed to room air $(n=3)$, respectively. These data confirm that hypoxic conditions were present in the solutions bubbled with argon gas. Reagents were then added to the solutions to initiate the experiment. The solutions were incubated for 1 hour while protected from light exposure. Hypoxic solutions were continually bubbled with argon gas during this period. Normoxic solutions were exposed to room air $\left(21 \% \mathrm{O}_{2}\right)$ under similar conditions. After the incubation, solutions were immediately distributed into a 96-well plate and read on a microplate fluorimeter.

\section{Experiments with Cells}

These experiments were performed on 96-well plates with each treatment group consisting of a separate row across the plate. Solutions were added to the plate one column at a time so that all treatment groups were loaded simultaneously, using an 8-channel 100 $\mu$ l Octapette (Corning-Costar).

\section{Materials and Reagents}

All fluoroprobes were purchased from Molecular Probes. All chemical reagents and drugs were purchased from Sigma (St. Louis, MO), except for myxothiazol, which was purchased from Fluka (Ronkonkoma, NY). Drugs were solubilized as stock solutions, as follows: $\mathrm{H}_{2}$ DCF-DA (5 mm), DHR (10 mm), DHE (5 mM), and R123 (5 mM) were solubilized in dimethylsulfoxide; antimycin A $(5 \mathrm{mg} / \mathrm{mL})$ and HQNO (10 $\mathrm{mm}$ ) were solubilized in ethanol; myxothiazol (10 
mм) was solubilized in methanol. The final concentration of the nonaqueous solvent never exceeded $1 \%$ in any experiment.

\section{References}

Al Mehdi AB, Shuman H, and Fisher AB (1997). Intracellular generation of reactive oxygen species during nonhypoxic lung ischemia. Am J Physiol 272:L294-L300.

Benov L, Sztejnberg L, and Fridovich I (1998). Critical evaluation of the use of hydroethidine as a measure of superoxide anion radical. Free Radic Biol Med 25:826-831.

Bindokas VP, Jordan J, Lee CC, and Miller RJ (1996). Superoxide production in rat hippocampal neurons: Selective imaging with hydroethidine. J Neurosci 16:1324-1336.

Brambilla L and Cantoni O (1998). Mitochondrial formation of hydrogen peroxide is causally linked to the antimycin A-mediated prevention of tert-butylhydroperoxide-induced U937 cell death. FEBS Lett 431:245-249.

Carter WO, Narayanan PK, and Robinson JP (1994). Intracellular hydrogen peroxide and superoxide anion detection in endothelial cells. J Leukoc Biol 55:253-258.

Cathcart R, Schwiers E, and Ames BN (1983). Detection of picomole levels of hydroperoxides using a fluorescent dichlorofluorescein assay. Anal Biochem 134:111-116.

Cutaia M, Parks N, Rounds S, Kroczynski J, and Tollefson K (2000). The role of the $\mathrm{Na}^{+} / \mathrm{H}^{+}$antiport in the $\mathrm{pH}$-dependent loss of cell viability ("pH paradox") following a metabolic insult in human pulmonary artery endothelial cells. Am J Physiol 278:L536-L544.

Dawson TL, Gores GJ, Nieminen AL, Herman B, and Lemasters JJ (1993). Mitochondria as a source of reactive oxygen species during reductive stress in rat hepatocytes. Am J Physiol 264:C961-C967.

Dugan LL, Sensi SL, Canzoniero LMT, Handran SD, Rothman SM, Lin TS, and Goldberg MP (1995). Mitochondrial production of reactive oxygen species in cortical neurons following exposure to N-methyl-d-aspartate. J Neurosci 15:63776388.

Garcia-Ruiz C, Colell A, Paris R, and Fernandez-Checa JC (2000). Direct interaction of GD3 ganglioside with mitochondria generates reactive oxygen species followed by mitochondrial permeability transition, cytochrome $c$ release, and caspase activation. FASEB J 14:847-858.
Haugland RP, editor (1996). Probes for reactive oxygen species, including nitric oxide. In: Handbook of fluorescent probes and research chemicals, 6th ed. Eugene, OR: Molecular Probes, 483-502.

Hempel SL, Buettner GR, O'Malley YQ, Wessels DA, and Flaherty DM (1999). Dihydrofluorescein diacetate is superior for detecting intracellular oxidants: Comparison with 2'7'dichlorodihydrofluorescein diacetate, and dihydrorhodamine 123. Free Radic Biol Med 27:146-159.

Kalinich JF, Ramakrishnan N, and McClain DE (1997). The antioxidant Trolox enhances the oxidation of $2^{\prime}, 7^{\prime}$ dichlorofluorescin to $2^{\prime}, 7^{\prime}$-dichlorofluorescein. Free Radic Res 26:37-47.

Lander HM (1997). An essential role for free radicals and derived species in signal transduction. FASEB J 11:118-124.

Marchesi E, Rota C, Fann YC, Chignell CF, and Mason RP (1999). Photoreduction of the fluorescein dye 2'7'dichlorofluorescein: A spin trapping and direct electron spin resonance study with implications for oxidative stress measurements. Free Radic Biol Med 26:148-161.

Martin BD, Schoenhard JA, and Sugden KD (1998). Hypervalent chromium mimics reactive oxygen species as measured by the oxidant-sensitive dyes 2'7'-dichlorofluorescin and dihydrorhodamine. Chem Res Toxicol 11:1402-1410.

Rota C, Fann YC, and Mason RP (1999). Phenoxyl free radical formation during the oxidation of the fluorescent dye $2^{\prime} 7^{\prime}$-dichlorofluorescein by horseradish peroxidase: Possible consequences for oxidative stress measurements. J Biol Chem 274:28161-28168.

Royall JA and Ischiropoulos H (1993). Evaluation of 2', 7 '-dichlororfluorescin and dihydrorhodamine 123 as fluorescent probes for intracellular $\mathrm{H}_{2} \mathrm{O}_{2}$ in cultured endothelial cells. Arch Biochem Biophys 302:348-355.

Slater EC (1973). The mechanism of action of the respiratory inhibitor, antimycin. Biochim Biophys Acta 301:129-154.

Turrens JF (1997). Superoxide production by the mitochondrial respiratory chain. Biosci Rep 17:3-8.

Zhu H, Bannenberg GL, and Moldeus P (1994). Oxidation pathways for the intracellular probe 2', 7'-dichlororfluorescin. Arch Toxicol 68:582-587.

Zulueta JJ, Sawhney R, Yu FS, Cote CC, and Hassoun PM (1997). Intracellular generation of reactive oxygen species in endothelial cells exposed to anoxia-reoxygenation. Am J Physiol 272:L897-L902. 